\title{
UNA MIRADA A LA EFECTIVIDAD DE LOS PROFESORES EN CHILE*
}

\author{
Bernardo Lara, Alejandra Mizala \\ y Andrea Repetto
}

\begin{abstract}
Este trabajo mide la correlación entre el resultado de los escolares en la prueba SIMCE de cuarto básico y las características observables de los docentes, sus prácticas en el aula y sus percepciones respecto de las capacidades de los alumnos. El análisis busca explicar el desempeño de los niños en base a sus propias características, las de sus familias, establecimientos y profesores, intentando corregir por sesgos en la asigna-
\end{abstract}

Bernardo Lara. Magíster en Economía Aplicada (CEA) e ingeniero civil industrial, Universidad de Chile. Fue ayudante de investigación del CEA, período en el cual participó en la realización de este trabajo. Actualmente es estudiante de economía de la educación en la Universidad de Stanford (EE.UU.).

Alejandra Mizala. Ph D. en Economía, Universidad de California, Berkeley. Directora del Centro de Economía Aplicada (CEA), Depto. Ingeniería Industrial, y directora académica del Centro de Investigación Avanzada en Educación, ambas instituciones de la Universidad de Chile.

Andrea Repetto. Doctora en Economía, Massachusetts Institute of Technology (MIT). Magíster en Economía e ingeniera comercial, Universidad Católica de Chile. Académica de la Escuela de Gobierno de la Universidad Adolfo Ibáñez (Santiago).

* Agradecemos a la oficina del SIMCE en el Ministerio de Educación por el acceso a los datos, y a Harald Beyer, Francisco Gallego, Francisco Lagos y a los asistentes al seminario realizado en el CEP el 15 de junio de 2010, en el que se presentó una primera versión de este trabajo, por sus sugerencias y comentarios. Este trabajo ha recibido el apoyo financiero de Fondecyt \# 1070316 y de CONICYT bajo el Proyecto CIE-05 Centro de Investigación Avanzada en Educación. 
ción de docentes tanto a través de escuelas como al interior de ellas. Los resultados muestran una correlación relevante entre resultados en el SIMCE y el género del profesor, su certificación como docente y sus años de experiencia. También muestran que existen prácticas simples al interior del aula que se correlacionan con un mejor desempeño escolar. Finalmente, los resultados sugieren que aquellos profesores que creen más en la capacidad de sus alumnos tienen estudiantes con mejores rendimientos en las pruebas estandarizadas.

Palabras clave: experiencia docente; desempeño escolar; prácticas pedagógicas.

Clasificación JEL: I20, I21.

Recibido: junio 2010. Aceptado: septiembre 2010.

\section{Introducción}

$\mathrm{E}_{1}$ debate internacional sobre cómo elevar la calidad de la educación se ha centrado en el rol de los distintos participantes del proceso educativo, incluyendo tanto a las familias como a las escuelas, las autoridades y, en particular, los profesores. Dado que la mayor parte del proceso que viven los niños al interior de los establecimientos escolares pasa por sus profesores, la importancia de los docentes es innegable. Sin embargo, no existe consenso en la literatura en relación a qué fracción de la variabilidad observada en los resultados escolares se debe a diferencias en los docentes que los educan, ni tampoco sobre los efectos de características observables de los profesores en el aprendizaje de los niños, o sobre las prácticas específicas que definen una buena labor docente en el aula.

Chile no ha estado ajeno a este debate. El atractivo para los jóvenes que egresan de la enseñanza secundaria de seguir la carrera docente, el proceso formativo al interior de las facultades de educación, el sistema de remuneraciones y evaluación de los docentes, y la valoración social de estos profesionales, son todos temas de análisis entre académicos y entre quienes diseñan, definen e implementan las políticas educacionales en el país.

La literatura sobre el rol de los docentes en el desempeño escolar de los niños en Chile es, sin embargo, escasa, en buena parte debido a 
la falta de información que ligue los resultados académicos de los alumnos con las características de sus profesores. Este trabajo intenta medir el efecto que características observables de los docentes tienen sobre el resultado de los escolares en la prueba SIMCE (Sistema de Medición de la Calidad de la Educación) de cuarto básico. La disponibilidad de una encuesta docente levantada por el Ministerio de Educación que reúne información sobre las credenciales académicas de los profesores y su manera de abordar el proceso de enseñanza, y que a la vez identifica las salas y colegios en los que se desempeñan, es lo que nos permite estimar estas correlaciones.

El análisis se centra en los alumnos que rindieron el SIMCE de cuarto básico en el año 2002. El foco en este nivel responde al hecho de que un solo profesor de aula está encargado de enseñar tanto matemáticas como lenguaje, áreas lectivas en las que centramos el análisis. Sin embargo, los datos con los que contamos representan un corte transversal. Esto dificulta la corrección por los sesgos asociados a la elección de colegios por parte de las familias y de los profesores, y a la selección de alumnos y docentes por parte de los establecimientos educacionales ${ }^{1}$. Tampoco permiten hacer un análisis de valor agregado. Por tanto, y si bien realizamos correcciones que permiten hacernos cargo de estas limitaciones estadísticas, los resultados pueden ser entendidos alternativamente como una descripción de la correlación entre las características de los docentes y los rendimientos académicos de los niños que ellos educan, más que como una relación causal desde las características de los docentes a los puntajes obtenidos por los niños en el SIMCE.

Nuestro análisis consta de tres partes. En la primera describimos los rasgos generales de los profesores en Chile en todos los niveles del sistema escolar. El análisis revela que, al igual que en otros países, los profesores son en su mayoría mujeres. Asimismo, los datos muestran que la mayor parte posee un título profesional en educación y que un amplio porcentaje tiene más de 20 años de experiencia en el aula.

La segunda parte de nuestro análisis busca explicar las diferencias en el desempeño de los niños en el SIMCE 2002 en base a diferencias en sus propias características y en las de sus familias, los establecimientos y sus profesores. Para corregir por sesgos en la asignación de profesores a través de escuelas, controlamos por el desempeño de los estableci-

\footnotetext{
${ }^{1}$ Véase Lara, Mizala y Repetto (2009) para una discusión reciente.
} 
mientos en pruebas SIMCE anteriores. A su vez, para corregir por sesgos en la asignación de profesores al interior de las escuelas, centramos el trabajo en los colegios que tienen una sola sala en el nivel.

Nuestros resultados indican que en la prueba de lenguaje - no así en matemáticas - los alumnos educados por docentes mujeres obtienen mejores resultados en el SIMCE que aquellos educados por docentes hombres. Asimismo, en ambas pruebas existe correlación entre el desempeño escolar y que el profesor tenga un título docente, aunque el tipo de institución - universidad o escuela normal—que confirió el título sólo es relevante en la prueba de lenguaje. Este efecto es económicamente relevante: los alumnos cuyos docentes poseen el título de profesor obtienen un puntaje adicional de hasta $13 \%$ de una desviación estándar. Finalmente, el efecto de los años de experiencia docente es no lineal, fundamentalmente en lenguaje, con las mayores ganancias en los primeros años de carrera profesional, resultado coincidente con el de investigaciones para otros países.

En esta parte del trabajo también se analizan las prácticas que los profesores declaran realizar en su labor docente, las preocupaciones que éstos declaran y el grado de satisfacción con su trabajo. Nuestro análisis revela que prácticas tan simples como leer textos y realizar ejercicios de comprensión lectora y llevar a los niños a la biblioteca se correlacionan con un mejor desempeño en el SIMCE de lenguaje. Además, aquellos profesores que declaran creer que sus alumnos cursarán educación superior obtienen un puntaje adicional de un $12 \%$ de una desviación estándar en ambas pruebas SIMCE. Asimismo, los profesores de las salas de mejor desempeño tienden a declarar con mayor frecuencia que creen que sus alumnos cursarán educación superior. De igual forma, también tienden a declarar que desarrollan actividades específicas como, por ejemplo, pedir a los alumnos que expliquen a sus compañeros cómo realizaron las tareas y plantear situaciones y problemas cotidianos en matemáticas, así como acudir a la biblioteca y realizar ejercicios de comprensión lectora en lenguaje. Estos resultados, sin embargo, deben leerse con cuidado, debido a que estas prácticas pedagógicas simplemente pueden estar correlacionadas con otras variables no observables. Es posible, asimismo, que la causalidad sea inversa, de modo que niños con buenas características no observables pueden permitir un mejor desarrollo de la clase, favoreciendo la realización de ciertas actividades docentes. 
Los resultados de nuestro análisis son consistentes con lo que obtienen estudios realizados para otros países que cuentan con mejores datos. También son consistentes con las prácticas pedagógicas a las que aluden directores de escuelas de distinta efectividad entrevistados por Henríquez et al. (2009). Asimismo, contienen información útil para las políticas respecto de los profesores. Por ejemplo, sugiere que los efectos más relevantes en términos de credenciales de formación se relacionan con tener un título más que con si el profesor se formó en una universidad o en una escuela normal, en particular en matemáticas, pero no así en lenguaje. Los resultados también sugieren la importancia de que el docente crea en la capacidad de sus alumnos y de que existan ciertas prácticas al interior de la sala de clases que se correlacionan con un mejor desempeño de los niños en el SIMCE.

El trabajo está organizado de la siguiente forma. La sección II describe las características de los profesores en Chile. La sección III analiza la literatura internacional sobre el rol de los docentes en el aprendizaje escolar. La sección que sigue presenta nuestros datos y metodología de estimación, y discute sus limitaciones. La sección V analiza las diferencias en el desempeño de los niños en el SIMCE 2002 en base a diferencias en sus propias características y en las de sus familias, los establecimientos y sus profesores. Esta sección también indaga acerca de la relación entre los resultados del SIMCE y las prácticas que los profesores reportan realizar en el aula; asimismo, correlaciona la probabilidad de ser un profesor dentro del $25 \%$ superior con las actividades y preocupaciones que ellos declaran. Finalmente, la sección VI presenta nuestras conclusiones.

\section{II. ¿Quiénes son los profesores en Chile y cómo se les remunera?}

De acuerdo a las estadísticas del Ministerio de Educación, en el año 2002 había 140.774 profesores en el país ${ }^{2}$. De ellos, 56\% trabajaba en colegios municipales, $29,3 \%$ en colegios particulares subvencionados y el restante $12,9 \%$ en colegios particulares pagados. Asimismo, $86 \%$ de los profesores se desempeñaba en colegios urbanos. En términos de niveles educacionales, 59\% se desempeñaba en la educación

${ }^{2}$ Nos referimos al 2002 porque es el año cubierto por el SIMCE y la encuesta docente que analizamos en el resto del trabajo. A continuación mostramos que no ha habido grandes cambios en las características medias de los profesores en el tiempo. 
básica, $27 \%$ en la educación media y 8,3\% en la educación preescolar. El 85,5\% eran docentes de aula, el 3,5\% directores, el 5,8\% directivos y el 3,6\% personal técnico-pedagógico.

La educación es una profesión feminizada: el $70 \%$ de los profesores son mujeres, que están concentradas en la educación preescolar y básica. En cambio, en la educación secundaria la participación de hombres y mujeres se vuelve más pareja. A pesar del alto porcentaje de mujeres profesoras, $51 \%$ de los cargos directivos son ocupados por hombres, algo similar a lo que ocurre con los directivos del Colegio de Profesores.

En relación a su educación y experiencia, la gran mayoría $(89,5 \%)$ de los profesores tenía un título universitario en el 2002. Casi un tercio de los docentes $(30,8 \%)$ tenía menos de 10 años de experiencia, mientras que el $24 \%$ tenía entre 10 y 19 años de experiencia, y el $45 \%$ más de 20 años en la docencia. Casi un tercio de los docentes tenía entre 40 y 50 años de edad y un poco más del $25 \%$ entre 50 y 60 años en 2002; sólo 9\% tenía menos de 30 años de edad.

$\mathrm{Al}$ igual que en la mayoría de los países, los profesores trabajan menos horas que el trabajador promedio. Sólo el $25 \%$ de los docentes trabajaba 44 horas a la semana en el año 2002 —éste es el número máximo de horas que se puede trabajar con un mismo empleador en el sistema subvencionado. El 26,5\% trabajaba entre 31 y 43 horas, y el $48,4 \%$ trabajaba 30 horas o menos. La gran mayoría $(85,6 \%)$ de los docentes se desempeñaba sólo en un colegio, y a esa fecha el $89,5 \%$ de ellos tenía un título en educación ${ }^{3}$.

El Gráfico $\mathrm{N}^{0} 1$ compara las características de los profesores del año 2002 con las de los profesores del año 2008. En general el perfil es muy similar, excepto por el aumento del número de profesores que se desempeñan en el sector particular subvencionado $(41,9 \%)$ en relación con el sector municipal $(45,4 \%)$. Esta situación se explica por el incremento que ha experimentado en los últimos años la matrícula escolar en los colegios particulares subvencionados y la consecuente disminución de la matrícula municipal. También se observa un mayor porcentaje de profesores que trabajan entre 31 y 43 horas (de 26,5\% a 39\%), posiblemente debido a la jornada escolar completa, y un aumento de los profesores con menos de 10 años de servicio (de $30,8 \%$ a $38,6 \%$ ), lo que está asociado a un mayor porcentaje $(16,5 \%)$ de profesores jóvenes menores de 30 años en el 2008.

${ }^{3}$ En el 2002 la jornada laboral establecida en el Código Laboral era de 48 horas semanales, la que a partir del 2005 se redujo a 45 horas semanales. 


\section{GRÁFICO No 1: CARACTERÍSTICAS DE LOS DOCENTES 2002 Y 2008 (\%)}

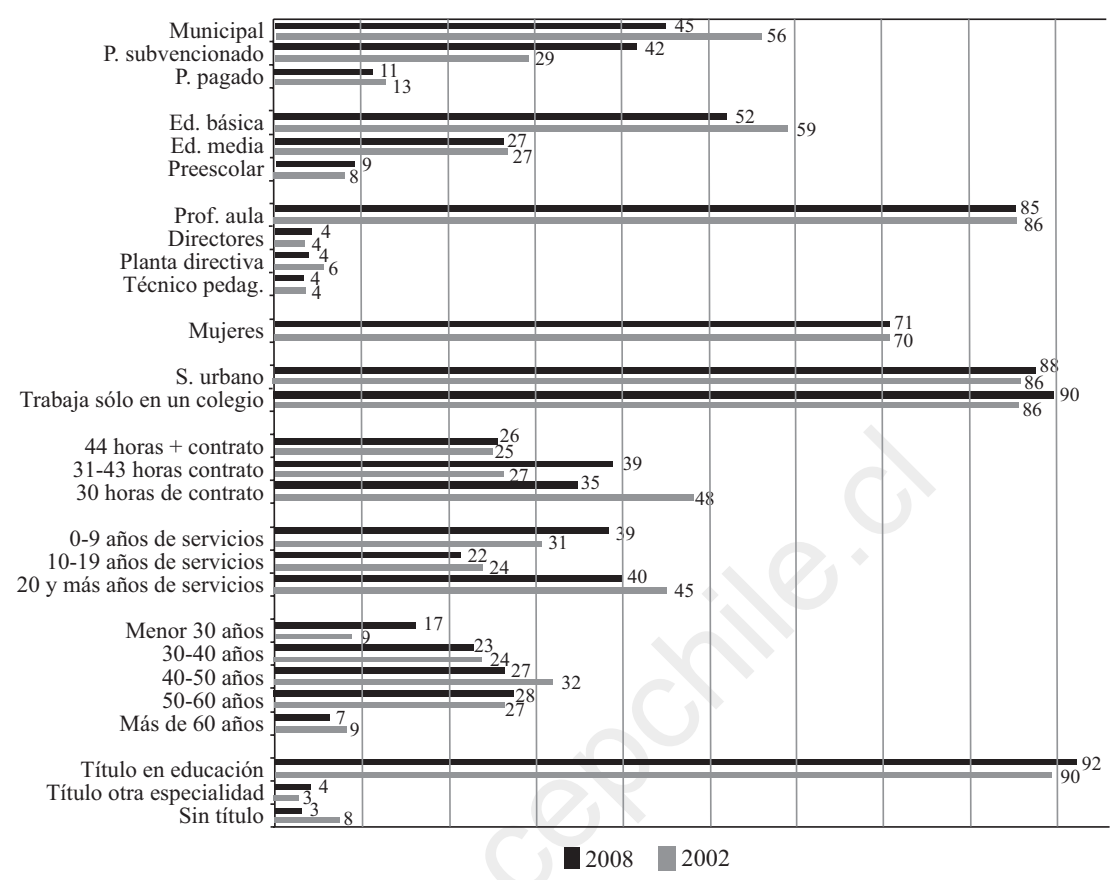

Fuente: Ministerio de Educación, División de Planificación y Presupuesto, Estadísticas de la Educación 2002 y 2008.

Los datos anteriores son consistentes con los obtenidos a partir de la encuesta aplicada a más de 6.000 profesores de lenguaje y matemáticas de $4^{\circ}$ básico, que se realizó en conjunto con la prueba SIMCE del año 2002, y que analizamos más adelante. Ella muestra que la edad promedio de los encuestados era 45,7 años, con 20,9 años promedio de experiencia. El 95\% de ellos tenía título y el 76\% tenía título universitario. Esta encuesta también indaga acerca del grado de satisfacción de los docentes con su trabajo. Llama la atención la alta satisfacción que los docentes declaran tener con su labor: el 99\% reporta que disfruta de sus clases y un porcentaje similar dice que sus alumnos los respetan. Asimismo, el 97\% dice tener libertad para tomar decisiones y el $91 \%$ reporta que el director del colegio reconoce su trabajo.

Respecto de las remuneraciones, en las últimas dos décadas los salarios de los profesores han experimentado un aumento importante 
en el país. De hecho, entre los años 1990 y 2009 los salarios reales de los docentes se incrementaron en 199\%, alza significativamente mayor que la experimentada por el índice general de remuneraciones en igual lapso de tiempo. Adicionalmente, en este período se incorporó una serie de incentivos a los docentes: el Sistema Nacional de Evaluación del Desempeño Docente de los Establecimientos Subvencionados (SNED), la Asignación de Excelencia Pedagógica (AEP) y la Asignación Variable por Desempeño Individual (AVDI) vinculada a la evaluación docente obligatoria de los profesores que trabajan en el sector municipal ${ }^{4}$.

\section{La literatura internacional}

Desde el estudio pionero de Coleman et al. (1966) - el Coleman Report- la estimación de funciones de producción educacionales ha intentado vincular resultados educativos con diversos insumos, entre ellos las características de los profesores. Una de las principales conclusiones del Coleman Report es que el determinante más relevante de los resultados académicos de los escolares es su nivel socioeconómico; el aporte del colegio y los profesores sería más bien reducido. Sin embargo, un amplio grupo de estudios posteriores cuestiona metodológicamente estos resultados, indicando que el colegio sí jugaría un rol en los aprendizajes que en la práctica es estadísticamente difícil de identificar.

En efecto, existe un debate intenso en relación a los factores que afectan los resultados educativos. De hecho, muchos de los estudios llegan a resultados contradictorios debido a la falta de datos adecuados, a los supuestos utilizados y a problemas metodológicos que enfrentan, fundamentalmente la endogeneidad de las variables explicativas. Los resultados también varían dependiendo de las condiciones específicas de los colegios y de las variables de contexto incluidas en el análisis.

En el caso del rol de los profesores es importante tomar en cuenta que ellos no se distribuyen aleatoriamente a través de colegios y, posiblemente, tampoco al interior de los establecimientos. El impacto estimado de los profesores en el proceso educativo estaría sesgado si la asignación de alumnos y profesores en colegios y salas induce una correlación entre las características de los educadores y variables no observables que afectan el desempeño escolar. Por ejemplo, si los pro-

\footnotetext{
${ }^{4}$ Para más detalles véase Mizala y Schneider (2010).
} 
fesores mejor capacitados tienden a enseñar en los colegios a los que asisten los niños más aventajados — posiblemente porque los mejores profesores pueden optar por mejores condiciones laborales-, entonces un análisis simple llevaría a sobrestimar el efecto de los profesores en el rendimiento escolar.

La literatura basada en datos de corte transversal ha tenido dificultades para hacerse cargo de estos problemas. Una revisión se encuentra en Hanushek (1997). Sin embargo, el trabajo reciente de Clotfelter, Ladd y Vigdor (2006a) muestra cuán relevante es esta asignación no aleatoria utilizando datos administrativos para alumnos de quinto básico en el estado de Carolina del Norte. Si bien estos datos representan un corte transversal, la incorporación de efectos fijos por colegios y el análisis de una submuestra de establecimientos con características observables balanceadas de los alumnos en las distintas salas permiten corregir por sesgos tanto al interior del establecimiento como a través de ellos. Los resultados de Clotfelter et al. (2006a) sugieren que los sesgos más relevantes se refieren a la asignación de profesores a través de escuelas más que al interior de los establecimientos. Asimismo, el trabajo concluye que la experiencia de los profesores y su desempeño en exámenes de licenciatura son predictores robustos de los resultados escolares de sus alumnos.

Una literatura más reciente analiza el impacto de los profesores sobre los aprendizajes de los estudiantes utilizando datos de panel. Estos trabajos no sólo permiten seguir el desempeño de los niños en el tiempo, sino también identificar al docente que estaba a cargo de su educación en cada una de las etapas. Así, es posible identificar separadamente el rol que cumplen las habilidades de los alumnos, las características del profesor y las características de los establecimientos.

El estudio de Rivkin, Hanushek y Kain (2005) analiza datos para tres cohortes de estudiantes del estado de Texas y encuentra diferencias significativas entre colegios en términos del aprendizaje de sus alumnos. Asimismo, el trabajo sugiere que estas diferencias se explican fundamentalmente por el efecto de los profesores más que por diferencias en la organización de los colegios en liderazgo o en sus condiciones financieras. En particular, el efecto de la calidad de los profesores - asociado al efecto fijo de profesor- es mucho más fuerte que el efecto del tamaño de los cursos. Las estimaciones sugieren que las diferencias en la calidad de los profesores explican al menos el 7,5\% de la variación total en los resultados de los alumnos. 
Una limitación de esta literatura se refiere a la identificación de la calidad de los profesores con efectos fijos de docente. Si bien esta información es de interés para cuantificar la importancia de los docentes en explicar la varianza de los resultados escolares de los niños, no permite realizar propuestas de política que promuevan un mejor desempeño académico. La literatura posterior ha intentado identificar los efectos de características observables y modificables de los docentes a través de políticas e incentivos, diferenciándolos de su habilidad no medible.

El trabajo de Hanushek, Kain, O’Brien y Rivkin (2005) usa datos de panel de estudiantes y profesores de un distrito grande del estado de Texas para estimar las variaciones en la calidad de los profesores mediante un enfoque semiparamétrico basado en medidas de valor agregado del aprendizaje de los estudiantes. Los autores consideran el progreso en el aprendizaje de los estudiantes para estimar el valor agregado de los profesores, variable que utilizan como medida de su calidad. Las estimaciones confirman la existencia de una variación sustancial en la efectividad de los profesores — nuevamente medida por el efecto fijo de profesor-, mucha de la cual ocurre más bien al interior de los colegios y no entre los colegios. En efecto, el alumno promedio que tiene un profesor en el percentil 85 de calidad obtiene ganancias que superan en 0,22 desviaciones estándar el resultado que hubiese obtenido con el profesor de calidad mediana. Asimismo, los resultados permiten concluir que los buenos profesores tienen efectos positivos sobre todos los estudiantes que atienden, independientemente del nivel de habilidad de los alumnos. Un análisis complementario relaciona luego esta medida de calidad del docente con características medibles, como experiencia, certificación y grados académicos. De ellas, sólo la experiencia tiene un efecto estadísticamente significativo, con las mayores ganancias en los primeros años de trabajo docente.

Por su parte, Kukla-Acevedo (2009) obtiene resultados similares basados en datos para el estado de Kentucky. En adición, encuentra que el desempeño de los profesores en sus estudios universitarios y las horas dedicadas al estudio de la disciplina que imparten también son predictores de los resultados académicos de sus alumnos. Asimismo muestra que el efecto de las características de los profesores depende de sus años de experiencia como docentes, y que la experiencia también interactúa con características de los niños, como su nivel socioeconómico y su origen racial. 
Rockoff (2004) analiza un set de datos de panel de estudiantes que asisten a colegios de dos distritos del estado de New Jersey. Los datos permiten comparar el desempeño de los mismos estudiantes con diferentes profesores y por tanto distinguir la variación de la calidad de los profesores de la variación de la capacidad y habilidad de los estudiantes, y a la vez distinguir la calidad de los profesores de otras características como el tamaño del curso. El autor usa random-effects meta-analysis para medir la varianza en los efectos fijos de los profesores considerando explícitamente el error de estimación. Concluye que hay grandes diferencias en la calidad de los profesores al interior de los colegios: un aumento de una desviación estándar en la calidad de los profesores aumenta los resultados en las pruebas estandarizadas de lenguaje y matemáticas en 0,1 desviaciones estándar. También encuentra evidencia de que la experiencia de los profesores aumenta significativamente el logro de los estudiantes, particularmente en lectura. Los puntajes de las pruebas de lectura difieren, en promedio, en alrededor de 0,17 desviaciones estándar entre un profesor que está comenzando su carrera y uno con 10 o más años de experiencia.

Por su parte, Clotfelter, Ladd y Vigdor (2006b) analizan datos longitudinales de alumnos entre tercero y quinto año de primaria para un período de 10 años. Si bien la aproximación metodológica es similar a la de Hanushek et al. (2005) y a la de Rockoff (2004), la mayor riqueza de la información permite abordar preguntas adicionales. En particular, el estudio puede identificar si el efecto de una mayor experiencia docente se debe a que los mismos profesores se vuelven mejores con el tiempo, o a que los peores profesores se retiran de la carrera docente más temprano. También puede determinar el rol de la certificación de los profesores y corregir por la posibilidad de que los profesores de mejores calificaciones atiendan con mayor frecuencia a niños de mayor habilidad escolar. Los resultados indican que las mayores ganancias debido a la experiencia suceden en los primeros años de la carrera docente y que el efecto medio no se debe a un diferencial de atrición en profesores de distinta habilidad. Asimismo, se obtiene que los tests de licenciatura tienen poder predictivo de las habilidades de los profesores, y que el tipo de licencia y el proceso de certificación también son relevantes, en particular en matemáticas. El haber obtenido el título de profesor en una institución de alta calidad se relaciona también con un mejor desempeño de los niños. Por su parte, el efecto de poseer un postgrado es positivo, pero pequeño. 
Finalmente, Goldhaber y Brewer (2000) y Clotfelter et al. (2007) centran su análisis en la relevancia de los procedimientos de certificación y su rol en los resultados de sus alumnos. El requerimiento de licencias para enseñar, la relación entre salarios docentes y su certificación, y la asignación de docentes de acuerdo a sus credenciales académicas son todas políticas públicas con el potencial de afectar el desempeño de los niños si estos procesos de licenciamiento y certificación tienen un efecto causal sobre su aprendizaje. Clotfelter et al., usando datos de panel y efectos fijos por alumno para estudiantes de secundaria de Carolina del Norte, encuentran un efecto importante de la certificación de los docentes en las áreas en las que imparten conocimiento, con efectos de hasta 0,12 desviaciones estándar en el desempeño de los jóvenes. Los resultados de Goldhaber y Brewer (2000) confirman que los requerimientos de certificación tienen efectos relevantes sobre los resultados escolares de jóvenes en secundaria.

Para Chile existen pocos estudios que intenten medir el impacto de las características de los profesores en el aprendizaje de los estudiantes, en parte porque es difícil identificar adecuadamente el efecto de distintos insumos educativos sobre el aprendizaje, dado que no existen datos de panel que permitan parear a los estudiantes con sus profesores y seguir su desempeño en el tiempo. Recientemente, sin embargo, dos estudios han correlacionado los resultados de los alumnos en el SIMCE con los de los profesores en las evaluaciones docentes vigentes desde el 2002, descritas más arriba, utilizando datos de corte transversal. Esta característica de los datos representa una limitación importante. Así, sin correcciones por la no aleatoriedad en la asignación de profesores y niños, y también por el carácter voluntario de algunas de las evaluaciones docentes, los resultados deben leerse como la existencia de una correlación entre el desempeño de los alumnos y el de los profesores en las respectivas pruebas. Bravo et al. (2008) relacionan los resultados del programa voluntario AEP, de la evaluación docente obligatoria en escuelas municipales y del programa AVDI para profesores destacados, con los resultados individuales del SIMCE de cuarto básico de diversos años según el instrumento de evaluación. Por su parte, León, Manzi y Paredes (2009) relacionan los resultados de las evaluaciones docentes del 2005 y 2006 con los del SIMCE del 2006 a nivel de promedio de los colegios, usando la metodología del modelo lineal jerárquico. Ambos 
trabajos encuentran una correlación positiva entre el desempeño de los profesores y el de los niños en el SIMCE.

En adición a estos estudios, Toledo (2010) utiliza los SIMCE de $4^{\circ}$ básico del 2005 y 2006, y sigue el procedimiento de Clotfelter et al. (2006a) al introducir efectos fijos por colegio y utilizar una muestra de los datos en los que las variables observables de los alumnos son similares a través de salas. Esta última corrección resuelve el problema de la asignación no aleatoria al interior de los colegios, siempre que ella esté basada en variables observables. El trabajo encuentra un efecto positivo de las profesoras mujeres y un efecto no lineal de la experiencia, que se vuelve decreciente luego de los cinco años de trabajo docente.

En este trabajo utilizamos los datos individuales de corte transversal disponibles para el 2002. Nuestra metodología reconoce, como en Clotfelter et al. (2006a), la asignación no aleatoria de profesores y alumnos, tanto a través de los establecimientos como al interior de ellos. En efecto, primero incluimos como variable independiente el resultado del SIMCE del colegio en años anteriores para corregir por sesgos en la asignación de alumnos y profesores a través de colegios. Luego centramos el análisis en establecimientos en los que existe una sola sala de clases en el nivel de cuarto básico para así hacernos cargo de sesgos de asignación de docentes y niños al interior del colegio. En la sección que sigue discutimos nuestro método de estimación en mayor detalle.

\section{Datos y método}

Los datos utilizados en este trabajo provienen de la prueba SIMCE que se aplica a los alumnos de $4^{\circ}$ y $8^{\circ}$ básico y de $2^{\circ}$ de enseñanza media. Este trabajo utiliza la prueba que se aplicó a los alumnos de $4^{\circ}$ básico en el año 2002. Usamos datos de $4^{\circ}$ básico porque hasta este nivel los alumnos de enseñanza básica tienen un solo profesor; a partir de $5^{\circ}$ básico cada disciplina es impartida por un profesor especializado. Sin embargo, cabe destacar que el profesor de $4^{\circ}$ básico no necesariamente ha sido el profesor de aula en años anteriores de los alumnos cuyo SIMCE es analizado. Lamentablemente, los datos disponibles no permiten identificar a los profesores que atendieron a estos niños desde el inicio de su escolaridad.

Junto con los puntajes obtenidos por los estudiantes en las pruebas de lenguaje y matemáticas, utilizamos los resultados de la encuesta 
que responden los padres en el momento de la aplicación de la prueba SIMCE. Este cuestionario entrega información acerca de las características socioeconómicas de los hogares, las razones que tomaron en cuenta los padres al momento de escoger el colegio, las expectativas de los padres respecto del nivel educacional que alcanzará su hijo/a, algunas actividades que realizan los padres con el niño en la casa y el nivel de satisfacción de los padres con el establecimiento educacional. Asimismo, utilizamos los resultados de la encuesta que se aplicó en el momento de la prueba a los profesores de los cursos evaluados. Ella contiene preguntas relativas a su formación profesional, los contenidos que enseñó durante el año escolar, algunas prácticas pedagógicas llevadas a cabo y su nivel de satisfacción como docente en el establecimiento educativo.

Una vez que combinamos la información en una base de datos única, realizamos algunas modificaciones. En primer lugar, nos focalizamos en los colegios que reciben subvención del Estado, ya que los colegios particulares pagados atienden a la elite del país y funcionan bajo condiciones muy diferentes. De hecho, sus profesores tienen otras condiciones laborales y salarios significativamente más elevados que el resto de la profesión. Asimismo, sus establecimientos no atienden a más de un $7 \%$ de la matrícula total.

En segundo lugar, modificamos algunas variables para adaptarlas mejor a nuestro análisis. Por ejemplo, el cuestionario de padres reporta el nivel educacional más alto alcanzado por ellos. Estos niveles fueron traducidos a variables dummy que indican si el nivel educacional alcanzado es de educación básica, media o superior (estudios en centros de formación técnica, institutos profesionales o universidades). Los padres reportan, además, el rango donde se ubica el ingreso mensual del hogar (por ejemplo, entre $\$ 400.000$ y $\$ 500.000$ ). Estos rangos fueron reemplazados por el punto medio del intervalo 5 .

Finalmente, como explicamos más en detalle a continuación, limitamos el análisis a los alumnos que asisten a establecimientos con un solo curso por nivel.

El Cuadro $\mathrm{N}^{0} 1$ resume las estadísticas descriptivas de las variables utilizadas. El puntaje promedio de la prueba de matemática es 236 con una desviación estándar de 52,3, y el puntaje promedio de lenguaje

${ }^{5}$ Como la distribución del ingreso en Chile es más bien plana hasta el percentil 90, es de esperar que usar el punto medio del intervalo no lleva a sesgos relevantes en la definición del ingreso familiar. 


\section{CUADRO N ${ }^{\circ}$ 1: $\quad$ ESTADÍSTICAS DESCRIPTIVAS}

\section{Alumno y su familia}

Puntaje matemáticas

Media

235.877

Desv. est.

(52.336)

$\mathrm{N}$

32248

Puntaje lenguaje

Media

241.899

Desv. est.

(51.687)

$\mathrm{N}$

31945

Dummy madre con educ. media

Media

0.402

Desv. est.

$(0.490)$

$\mathrm{N}$

32248

Dummy madre con educ. sup

Media

0.118

Desv. est.

$\mathrm{N}$

32248

Ln ingreso familiar

Media

11.625

Desv. est.

(0.817)

$\mathrm{N}$

32248

Número de personas en el hogar

Media

4.876

Desv. est.

(1.766)

$\mathrm{N}$

32248

Dummy mujer

Media

0.485

Desv. est.

(0.500)

$\mathrm{N}$

32248

Expectativas educ. superior

Media

0.451

Desv. est.

(0.498)

$\mathrm{N}$

32248

\section{Establecimiento}

Número de alumnos sala

Media

Desv. est.

$\mathrm{N}$

(11.921)

Media

2026

Ln matrícula

Desv. est.

5.226

$\mathrm{N}$

(0.771)

Madres con ed. sup. en el colegio

Media

0.090

Desv. est.

(0.137)

$\mathrm{N}$

$\mathrm{N}^{\mathrm{o}}$ colegios comuna

Media

57.614

Desv. est.

(45.483)

$\mathrm{N}$

2026

SIMCE matemáticas 1999 establecimiento

Media

236.661

Desv. est.

(24.976)

$\mathrm{N}$

2026

SIMCE lenguaje 1999 establecimiento

Media

235.004

Desv. est.

(25.891)

$\mathrm{N}$

2026

Dummy rural

Media

0.515

Desv. est.

$(0.500)$

$\mathrm{N}$

2026 


\section{Docentes}

Profesora mujer

Media

0.769

Desv. est.

(0.421)

$\mathrm{N}$

Edad profesor

Media

45.047

Desv. est.

(9.757)

$\mathrm{N}$

Experiencia profesor

Media

19.829

Desv. est.

(10.786)

$\mathrm{N}$

Profesor con título

Media

Desv. est.

$\mathrm{N}$

Profesor estudió en la universidad

Media

0.797

Desv. est.

$\mathrm{N}$

Profesor estudió en esc. normal

Media

0.145

Desv. est.

$\mathrm{N}$

\section{Docentes: Prácticas pedagógicas}

$\mathrm{Al}$ inicio de las clases el profesor escribe en la pizarra

Media

0.278

el título del contenido que estudiarán ese día

Desv. est.

(0.448)

$\mathrm{N}$

Al inicio de las clases el profesor relaciona los contenidos con los de clases pasadas

Media

0.778

Desv. est.

$(0.416)$

$\mathrm{N}$

Al inicio de las clases el profesor hace una pregunta para

Media

0.398

introducir el contenido

Desv. est.

$(0.490)$

$\mathrm{N}$

2026

Matemáticas: Hace que desarrollen cálculos mentales

Media

0.588

Desv. est.

(0.492)

$\mathrm{N}$

1805

Matemáticas: Da tareas más de 2 veces a la semana

Media

0.746

Desv. est.

$(0.435)$

$\mathrm{N}$

Matemáticas: Organiza juegos o competencias entre alumnos Media

Desv. est.

$(0.458)$

$\mathrm{N}$

1808

Matemáticas: Pide a los alumnos que expliquen a otros

Media

0.451

cómo realizaron la tarea

Desv. est.

$(0.498)$

$\mathrm{N}$

Matemáticas: Usa la vida cotidiana (plantea situaciones

Media

0.925

y/o problemas)

Desv. est.

$\mathrm{N}$

Lenguaje: Da tareas más de 2 veces a la semana

Media

0.724

Desv. est.

$\mathrm{N}$

Lenguaje: Hace leer textos y ejercitar la comprensión lectora Media

Desv. est. 


\begin{tabular}{|c|c|c|}
\hline Lenguaje: Hace redactar pequeños textos literarios & $\begin{array}{l}\text { Media } \\
\text { Desv. est. } \\
\text { N }\end{array}$ & $\begin{array}{r}0.649 \\
(0.477) \\
1847\end{array}$ \\
\hline Lenguaje: Hace que los alumnos realicen dramatizaciones & $\begin{array}{l}\text { Media } \\
\text { Desv. est. } \\
\text { N }\end{array}$ & $\begin{array}{r}0.244 \\
(0.429) \\
1846\end{array}$ \\
\hline Lenguaje: Hace que los alumnos acudan a la biblioteca & $\begin{array}{l}\text { Media } \\
\text { Desv. est. } \\
\text { N }\end{array}$ & $\begin{array}{r}0.287 \\
(0.452) \\
1816\end{array}$ \\
\hline Le preocupa la disciplina & $\begin{array}{l}\text { Media } \\
\text { Desv. est. } \\
\text { N }\end{array}$ & $\begin{array}{r}0.147 \\
(0.354) \\
1998\end{array}$ \\
\hline $\begin{array}{l}\text { Se preocupa de los que quedan atrás y/o de que el } \\
\text { curso entienda }\end{array}$ & $\begin{array}{l}\text { Media } \\
\text { Desv. est. } \\
\text { N }\end{array}$ & $\begin{array}{r}0.926 \\
(0.262) \\
1998\end{array}$ \\
\hline $\begin{array}{l}\text { Propone actividades desafiantes y/o desafíos a los más } \\
\text { avanzados }\end{array}$ & $\begin{array}{l}\text { Media } \\
\text { Desv. est. } \\
\text { N }\end{array}$ & $\begin{array}{r}0.328 \\
(0.470) \\
1998\end{array}$ \\
\hline $\begin{array}{l}\text { Se preocupa de que todos realicen actividades y/o de mot } \\
\text { para que trabajen }\end{array}$ & $\begin{array}{l}\text { Media } \\
\text { Desv. est. } \\
\mathrm{N}\end{array}$ & $\begin{array}{r}0.856 \\
(0.351) \\
1998\end{array}$ \\
\hline Alcanza a pasar todos contenidos y/o actividades & $\begin{array}{l}\text { Media } \\
\text { Desv. est. } \\
\text { N }\end{array}$ & $\begin{array}{r}0.057 \\
(0.232) \\
1998\end{array}$ \\
\hline Cree que los alumnos cursarán la educación superior & $\begin{array}{l}\text { Media } \\
\text { Desv. est. } \\
\text { N }\end{array}$ & $\begin{array}{r}0.362 \\
(0.481) \\
2026\end{array}$ \\
\hline Se declara muy satisfecho con su trabajo & $\begin{array}{l}\text { Media } \\
\text { Desv. est. } \\
\text { N }\end{array}$ & $\begin{array}{r}0.007 \\
(0.083) \\
2026\end{array}$ \\
\hline
\end{tabular}

es 242 con una desviación estándar de 51,7. El 49\% de los estudiantes son mujeres, el $40 \%$ de las madres tienen educación media y el $12 \%$, educación superior. El $45 \%$ de los padres espera que su hijo/a curse educación superior. En promedio, cada sala de clases tiene 24 estudiantes, con una desviación estándar de 12 alumnos, y el $9 \%$ de las madres a nivel del colegio tiene educación superior.

En relación a las estrategias pedagógicas declaradas por los docentes $^{6}$, el $28 \%$ indica escribir el título del contenido de la clase en la pizarra al inicio de ésta; el $78 \%$ dice relacionar los contenidos que introducirá con los de las clases pasadas; el $40 \%$ reporta hacer una pregunta para introducir los contenidos; el 79\% dice preocuparse de que el curso vaya entendiendo los contenidos, y el $15 \%$ declara que una de sus mayores preocupaciones es mantener la disciplina. En matemáticas,

\footnotetext{
${ }^{6}$ Se consideran sólo las respuestas "muy frecuentemente" para a cada actividad.
} 
el $75 \%$ de los profesores dice dar tareas más de dos veces por semana; el $45 \%$ declara pedir a los alumnos que expliquen cómo hicieron sus tareas, el 30\% reporta organizar juegos y competencias en las clases y el $93 \%$ usa la vida cotidiana para plantear situaciones o problemas a resolver. En lenguaje, el 24\% declara pedir a los alumnos realizar dramatizaciones; el 93\% hace leer textos y ejercitar comprensión lectora, y el $29 \%$ responde que acude a la biblioteca con los alumnos. Es posible que los docentes respondan lo que se espera de ellos, y no lo que realmente hacen en las clases. Aún así, las desviaciones estándar obtenidas en algunas variables permiten pensar que hay un grado interesante de heterogeneidad en las respuestas.

El uso de datos de corte transversal para estimar el efecto de los distintos insumos educacionales acarrea dificultades metodológicas para la estimación. Por una parte, no permite realizar análisis de valor agregado. El desempeño escolar de un niño en un año dado es el resultado de la historia de insumos escolares a los que ha estado expuesto - profesores, establecimientos escolares, recursos en el hogar, etc.- - además de sus habilidades innatas. Los datos con los que contamos no registran esta historia, ni tampoco tienen información sobre el desempeño en evaluaciones anteriores que pudiesen capturar de manera resumida los efectos de aquella historia de insumos. De hecho, la prueba que utilizamos es la primera prueba nacional que rinden estos niños a lo largo de su escolaridad. Esta limitante es importante y tiene el potencial de invalidar nuestros resultados en la medida en que los insumos pasados y las habilidades innatas estén correlacionados con las variables independientes incluidas en nuestro modelo ${ }^{7}$.

Asimismo, en la medida en que las variables observables estén correlacionadas con factores no cuantificables relevantes para el desempeño de los niños, los coeficientes estimados estarán sesgados. En el caso de los profesores, si los más hábiles, por ejemplo, son contratados por las escuelas a las que asisten los niños con mayor capacidad de aprendizaje, entonces se sobrestimará el efecto de las características de los docentes sobre el desempeño escolar medio de los niños. Esta asignación no aleatoria también puede suceder al interior de los colegios

${ }^{7}$ Sin embargo, Clotfelter et al. (2006a) corrigen por el puntaje de los niños en pruebas estandarizadas anteriores además de por efectos fijos de colegios. Ellos muestran que los resultados se mantienen prácticamente inalterados si esta variable se omite del análisis. 
si tanto niños como profesores son asignados en las distintas salas de acuerdo a sus habilidades.

Específicamente, estudiamos los resultados de los niños en el SIMCE 2002 de cuarto básico como una función de las características de los niños y sus familias, de los establecimientos donde se educan y sus profesores. Así, si $\mathrm{A}_{i j d}$ representa el puntaje SIMCE de lenguaje o matemáticas del niño $i$ en la escuela $j$ a cargo del profesor $d$, entonces

$$
A_{i j d}=\alpha+X_{i} \beta+S_{j} \gamma+T_{d} \delta+\varepsilon_{i j d}
$$

Así, de acuerdo al modelo, el resultado en el SIMCE de cada niño puede ser asociado a un vector $X$ de características del niño y su familia; un conjunto de variables que describen al establecimiento educacional y la sala asignada al alumno, $S$; un set de variables observables de los profesores, $T$; y un error aleatorio $\varepsilon$. El vector $\delta$ representa el vector de parámetros de interés.

Entre las variables relacionadas con los alumnos y sus familias, incorporamos una dummy que indica si el alumno es mujer, el logaritmo natural del ingreso del hogar, el número de personas en el hogar, una dummy de si la madre completó la educación media, una dummy de si la madre completó la educación superior y una dummy que indica si los padres esperan que el niño o niña alcance la educación terciaria. Respecto de las características del establecimiento y la sala, incluimos el logaritmo natural del número de alumnos matriculados, el número de colegios en la comuna donde está ubicado el establecimiento educacional, una dummy que indica si el colegio es rural, el SIMCE promedio de cuarto básico del colegio en la prueba de la misma materia de 1999, el porcentaje de alumnos cuyas madres completaron la educación superior y el número de alumnos en la sala de clases. Finalmente, las variables que incorporamos sobre los profesores son una dummy que indica si el profesor es mujer, una dummy que indica si el sexo del profesor y el del alumno es el mismo, los años de experiencia docente en el nivel y al cuadrado, la edad del profesor, una dummy de si posee título y dummies de si estudió en una universidad o en una escuela normal.

La asignación no aleatoria de alumnos y profesores en los colegios puede llevar a estimaciones sesgadas del impacto de las características de los docentes sobre el desempeño de los niños si ella introduce 
correlaciones entre las características incluidas en el análisis y variables no observables que afecten el rendimiento escolar. Para soslayar este problema, Clotfelter et al. (2006a) corrigen por un conjunto de efectos fijos de colegio. Así, la efectividad de los profesores se estima a partir de la variación en sus características al interior de los colegios. En nuestro análisis optamos por corregir por el SIMCE de 1999 de cuarto básico de lenguaje o matemáticas, según corresponda, a nivel del colegio para capturar estos efectos particulares a cada colegio. Esto es, los resultados de nuestras estimaciones deben ser entendidos como los efectos de las diversas variables dada una cierta calidad del establecimiento escolar.

Para corregir por la asignación no aleatoria de niños y profesores al interior del colegio, Clotfelter et al. (2006a) analizan una submuestra de colegios en los que los niños muestran características observables balanceadas a través de las salas. Sus resultados indican que la relevancia de este posible sesgo sería menor. En este trabajo tomamos una opción diferente para corregir por eventuales sesgos debidos al proceso de asignación de profesores y niños al interior de los establecimientos. En efecto, estimamos el modelo descrito sólo en la submuestra de colegios que tienen una sola sala de cuarto básico. Debido a su tamaño, estos establecimientos no cuentan con suficientes alumnos como para tener más de una sala y por tanto no necesitan definir un criterio de asignación de los alumnos. En adición, sólo un profesor dicta clases de matemáticas y lenguaje en el nivel de $4^{\circ}$ básico. El $62 \%$ del total de los colegios permanece en nuestra submuestra una vez excluidos los colegios que tienen más de una sala (o curso) de $4^{\circ}$ básico. De los colegios excluidos, el $65 \%$ tiene dos salas, el $25 \%$ tiene tres y el resto tiene cuatro o más. Sin embargo, dado que los establecimientos de la submuestra son más pequeños, sólo representan el $28 \%$ de la matrícula total de $4^{\circ}$ básico.

Nuestro método para corregir por eventuales sesgos de selección tiene ventajas y desventajas. Por una parte, la estrategia de Clotfelter et al. (2006a) está basada en el análisis de características observables de los alumnos al interior de cada sala. Sin embargo, si la asignación está basada en variables no observables, los sesgos descritos pueden permanecer. Nuestro método asegura la corrección de este problema estadístico. Por otro lado, sin embargo, nuestro análisis tiene limitaciones en términos de su validez externa. En otras palabras, en la medida en que los colegios pequeños, con una sola sala, sean distintos de los demás es- 
tablecimientos, las conclusiones de nuestro estudio no pueden aplicarse al universo de estudiantes ${ }^{8}$.

La metodología se hace cargo de sesgos que provienen de la manera en que se parean profesores y alumnos, tanto al interior como a través de establecimientos. Sin embargo, ello no asegura que no haya otras fuentes de correlación no observables entre los resultados de los niños y las características de sus profesores. Una posible fuente de correlación - y por tanto de sesgos - es el hecho de que los padres pueden escoger establecimientos escolares, al menos en ciertas comunas de Chile. Por ejemplo, si los padres más motivados escogen los establecimientos en los que enseñan profesores de más alta habilidad, los coeficientes estimados estarían sesgados. En ese caso sería posible reinterpretar nuestras estimaciones como que los padres motivados eligen para sus hijos colegios en los que los profesores poseen características superiores. Existe evidencia, sin embargo, de que los padres de hogares de menores ingresos, a esta altura de la escolaridad de sus hijos, favorecen la cercanía al escoger establecimientos educacionales (Gallego y Hernando, 2009). Asimismo, el 89\% de los niños en la base SIMCE 2002 asiste a un establecimiento en la misma comuna en la que reside. Si se restringe la muestra a los niños en la Región Metropolitana, zona geográfica donde está la mayor oferta educacional del país y donde los padres tienen mayores posibilidades de optar, el $79 \%$ de los alumnos de $4^{\circ}$ básico estudia en la comuna donde vive. Adicionalmente, entre los alumnos de colegios pequeños con una sala en el nivel, en los que se basa nuestro análisis, la fracción es del 76\% ${ }^{9}$. Si bien estos datos sugieren que los motivos de elección de establecimiento guardan relación con características más bien exógenas, reconocemos que ésta no es evidencia suficiente como para descartar la existencia de estos sesgos.

${ }^{8}$ También estimamos el modelo utilizando la muestra completa de alumnos. Los resultados, naturalmente, difieren de los que se obtienen utilizando la submuestra. Una de las fuentes de potenciales diferencias es justamente el sesgo que resulta de la distribución no aleatoria de alumnos y docentes al interior del colegio. Otra es la posibilidad de que los establecimientos que analizamos sean distintos en la manera como combinan insumos productivos para generar aprendizaje; esto es, que tengan validez externa limitada. Estos resultados no se incluyen en el texto, pero pueden ser solicitados a los autores.

${ }^{9}$ Alternativamente, del total de la submuestra, el 34\% estudia en un colegio rural; el 25\%, en la Región Metropolitana, y el 16\% y 17\%, en las dos otras regiones grandes del país, en las que hay una mayor oferta educacional relativa (la V y VIII regiones, respectivamente). Por otro lado, los alumnos de la submuestra viven en comunas en las que hay 19,6 establecimientos en promedio. 


\section{Resultados}

\section{Características de los docentes}

Los resultados para la submuestra de estudiantes analizada se encuentran en el Cuadro $\mathrm{N}^{0}$ 2. Los efectos estimados indican que los alumnos de profesoras mujeres obtienen mejores resultados que los alumnos de profesores hombres en la prueba de lenguaje, no así en matemáticas. Este efecto de género no se ve potenciado en el caso en que el niño y el profesor sean del mismo sexo.

El modelo de regresión permite que la experiencia del profesor tenga un efecto no lineal sobre el desempeño en el SIMCE. Si bien no todas las variables pueden tener siempre un efecto estadísticamente significativo, su estimación punto sugiere que las ganancias de la experiencia decrecen en el tiempo; este efecto es más claro en lenguaje que en matemáticas. Es así que como lo muestra el Gráfico № 2: los mayores beneficios de la experiencia en lenguaje se concentran al principio de la carrera docente y tienden a desaparecer poco después de los 30 años de experiencia. Hay que tener en consideración, sin embargo, que nuestros datos son un corte transversal y que estas conclusiones pueden confundir efectos debidos al paso del tiempo con efectos relativos a las cohortes cubiertas por la encuesta.

Finalmente, la posesión de un título terciario de profesor se relaciona con ganancias relevantes en el SIMCE tanto en lenguaje como en matemáticas. Los efectos son relevantes y alcanzan al 9\% y 13\% de una desviación estándar en la muestra en lenguaje y matemáticas, respectivamente. Este efecto es importante, mayor que la diferencia estimada entre el rendimiento de niños en colegios educados en colegios particulares subvencionados y el de aquellos educados en colegios municipales (Lara, Mizala y Repetto, 2009). Por su parte, la institución que otorgó ese título se relaciona con un efecto sólo en el caso del área de lenguaje, con 3,7 puntos adicionales en el SIMCE, o el 6,9\% de una desviación estándar. En matemáticas no hay efectos adicionales estadísticamente significativos de si fue una escuela normal la que formó al docente o una institución universitaria.

La edad del profesor tiene un efecto negativo sobre el rendimiento de los alumnos, aunque sólo en matemáticas este efecto es estadísticamente significativo. 
CUADRO No 2: CARACTERÍSTICAS DEL PROFESOR Y DESEMPEÑO EN EL SIMCE

\begin{tabular}{|c|c|c|}
\hline & Matemáticas & Lenguaje \\
\hline Profesor mujer & $\begin{array}{c}0.009 \\
(1.254)\end{array}$ & $\begin{array}{c}2.154 * \\
(1.229)\end{array}$ \\
\hline Edad profesor & $\begin{array}{l}-0.256 * * \\
(0.115)\end{array}$ & $\begin{array}{l}-0.144 \\
(0.100)\end{array}$ \\
\hline Experiencia & $\begin{array}{l}0.629 * * * \\
(0.215)\end{array}$ & $\begin{array}{l}0.549 * * * \\
(0.192)\end{array}$ \\
\hline Experiencia al cuadrado & $\begin{array}{l}-0.009 \\
(0.005)\end{array}$ & $\begin{array}{l}-0.009 * * \\
(0.004)\end{array}$ \\
\hline Mismo género & $\begin{array}{c}1.055 \\
(0.697)\end{array}$ & $\begin{array}{c}0.612 \\
(0.729)\end{array}$ \\
\hline Profesor posee título & $\begin{array}{l}6.944 * * * \\
(2.039)\end{array}$ & $\begin{array}{l}4.465 * * \\
(2.150)\end{array}$ \\
\hline Título universitario & $\begin{array}{c}2.201 \\
(1.310)\end{array}$ & $\begin{array}{c}3.734 * \\
(1.921)\end{array}$ \\
\hline Escuela normal & $\begin{array}{c}2.880 \\
(2.737)\end{array}$ & $\begin{array}{c}3.716 \\
(2.529)\end{array}$ \\
\hline Madre educ. media & $\begin{array}{l}3.944 * * * \\
(0.737)\end{array}$ & $\begin{array}{l}3.579 * * * \\
(0.686)\end{array}$ \\
\hline Madre educ. superior & $\begin{array}{l}2.435 * * \\
(1.091)\end{array}$ & $\begin{array}{l}3.215 * * * \\
(1.076)\end{array}$ \\
\hline Log ingreso hogar & $\begin{array}{l}1.723 * * * \\
(0.456)\end{array}$ & $\begin{array}{l}2.146^{* * *} \\
(0.445)\end{array}$ \\
\hline $\mathrm{N}^{\mathrm{o}}$ personas hogar & $\begin{array}{l}-0.001 \\
(0.003)\end{array}$ & $\begin{array}{c}0.002 \\
(0.003)\end{array}$ \\
\hline Mujer & $\begin{array}{c}0.075 \\
(0.697)\end{array}$ & $\begin{array}{l}2.959 * * * \\
(0.729)\end{array}$ \\
\hline Expectativas ed. superior & $\begin{array}{l}4.915 * * * \\
(0.713)\end{array}$ & $\begin{array}{l}4.519 * * * \\
(0.693)\end{array}$ \\
\hline $\mathrm{N}^{\circ}$ alumnos por sala & $\begin{array}{l}0.180 * * \\
(0.077)\end{array}$ & $\begin{array}{l}0.149 * * \\
(0.069)\end{array}$ \\
\hline Ln matrícula & $\begin{array}{c}0.694 \\
(1.426)\end{array}$ & $\begin{array}{l}-0.011 \\
(1.204)\end{array}$ \\
\hline Rural & $\begin{array}{l}2.721 * * \\
(1.317)\end{array}$ & $\begin{array}{l}6.329 * * * \\
1.261\end{array}$ \\
\hline$\%$ madres con educ. superior colegio & $\begin{array}{l}52.845 * * * \\
(4.464)\end{array}$ & $\begin{array}{l}51.663 \text { *** } \\
(4.249)\end{array}$ \\
\hline $\mathrm{N}^{\circ}$ de colegios municipio & $\begin{array}{l}-0.030 * * * \\
(0.010)\end{array}$ & $\begin{array}{l}-0.035 * * * \\
(0.010)\end{array}$ \\
\hline Puntaje SIMCE 99 & $\begin{array}{l}0.350 * * * \\
(0.025)\end{array}$ & $\begin{array}{l}0.387 * * * \\
(0.022)\end{array}$ \\
\hline Constante & $\begin{array}{l}107.185 * * * \\
(10.675)\end{array}$ & $\begin{array}{l}98.135 * * * \\
(9.846)\end{array}$ \\
\hline $\mathrm{R}^{2}$ & 0.111 & 0.125 \\
\hline $\mathrm{N}$ & 32248 & 32321 \\
\hline
\end{tabular}

Notas:

*** Significativo al 1\%.** Significativo al 5\%. * Significativo al 10\%.

Errores estándar ajustados por cluster a nivel de colegio. 


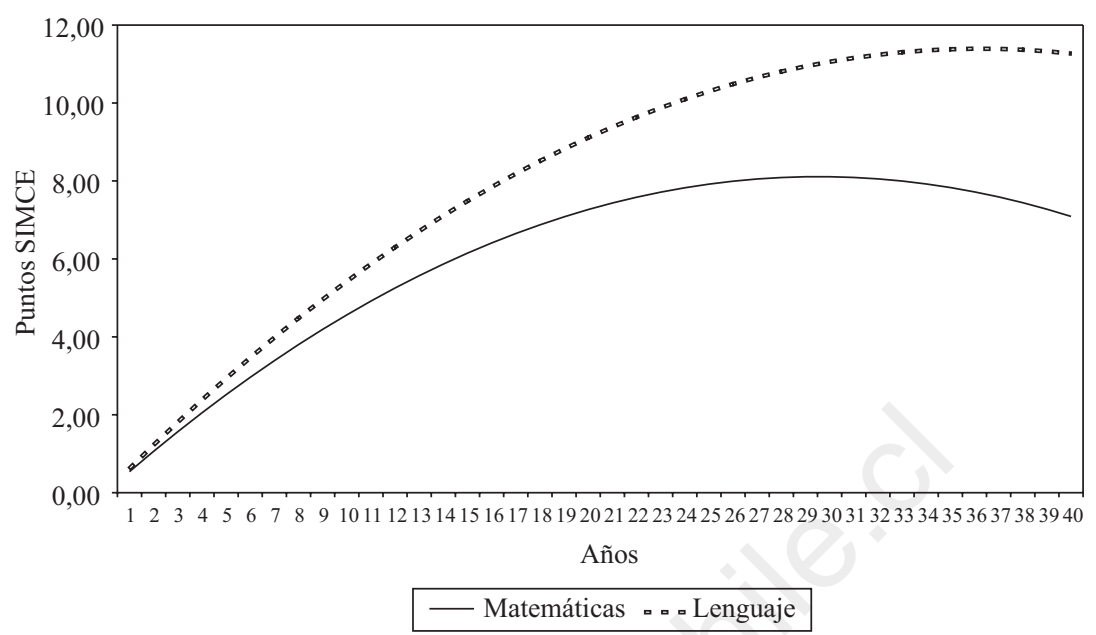

Prácticas en el aula

La encuesta docente no sólo recoge información sobre las características y credenciales de los profesores. También incluye una serie de preguntas sobre la manera en que los docentes enfrentan el proceso de enseñanza en el día a día. Específicamente, la encuesta levanta información sobre las actividades con las que un profesor comienza su clase, las actividades que realiza dentro y fuera del aula y sus principales preocupaciones mientras dicta una clase. Estas variables son posiblemente endógenas y probablemente están medidas con error. Asimismo, la escasa variabilidad observada en algunas respuestas sugiere que los profesores responden lo que se espera de ellos y no necesariamente lo que sucede al interior del aula. En esta subsección buscamos indagar si existe alguna correlación entre estas prácticas y preocupaciones, con el rendimiento de los niños en las pruebas SIMCE.

Con este fin incluimos en la ecuación (1) antes comentada las actividades y preocupaciones declaradas por los docentes. Consideramos una respuesta afirmativa cuando el profesor declara que muy frecuentemente realiza una determinada actividad o manifiesta una preocupa- 
ción. Las variables incluidas en el modelo son: desarrolla ejercicios de cálculo mental, organiza competencias o juegos matemáticos entre los alumnos, solicita a los alumnos que expliquen a sus compañeros cómo hicieron su tarea, plantea situaciones y problemas de la vida cotidiana, en el caso de matemáticas; para lenguaje, leer textos y realizar ejercicios de comprensión lectora, dar tareas más de dos veces por semana, redactar pequeños textos literarios, realizar dramatizaciones, acudir a la biblioteca del colegio. Asimismo, se incluyen variables que representan preocupaciones de los docentes, como mantener la disciplina, atender a los niños que se van quedando rezagados y que el curso entienda la materia, velar porque todo el curso realice las actividades y/o motivar para que trabajen, proporcionar actividades más desafiantes a los alumnos que avanzan más rápido y alcanzar a cubrir los contenidos programados para la clase. Finalmente, se incluyen variables que miden el grado de satisfacción con su trabajo, el grado de seguridad respecto de sus conocimientos acerca de las materias que enseña y si cree que sus alumnos cursarán educación superior. El Cuadro $\mathrm{N}^{\mathrm{o}} 3$ presenta estos resultados para lenguaje y matemáticas.

En el caso de matemáticas observamos que las prácticas docentes que se correlacionan positivamente con los resultados de los estudiantes en el SIMCE son desarrollar ejercicios de cálculo mental, preocuparse de los alumnos que van quedando rezagados, darles actividades más desafiantes a los alumnos más avanzados y pedir a los alumnos que expliquen a sus compañeros cómo hicieron su tarea, si bien ninguna de ellas es estadísticamente significativa. La preocupación por mantener la disciplina tiene un efecto negativo y significativo sobre el resultado en el SIMCE. En cambio el grado de seguridad del profesor respecto de las materias que enseña tiene un impacto positivo y significativo equivalente a $15 \%$ de una desviación estándar. También que el profesor crea que sus estudiantes van a cursar educación superior tiene un efecto positivo y significativo equivalente a $11 \%$ de una desviación estándar.

En el caso de la prueba de lenguaje, obtenemos que un número mayor de prácticas pedagógicas tiene una correlación estadísticamente significativa con buenos resultados en el SIMCE comparados con el caso de matemáticas. En particular, hacer a los niños acudir a la biblioteca y leer textos y realizar ejercicios de comprensión lectora. Nuevamente, los profesores cuyas salas tienen puntajes más altos en el SIMCE tien- 
CUADRO N ${ }^{\circ}$ 3: PRÁCTICAS PEDAGÓGICAS Y DESEMPEÑO EN EL SIMCE

\begin{tabular}{ll}
\hline & Matemática \\
\hline Desarrolla ejercicios de cálculo mental & 1.525 \\
& $(1.161)$ \\
Organiza competencias o juegos matemáticos entre los alumnos & -1.158 \\
& $(1.194)$ \\
Pide a los alumnos que expliquen a otros compañeros cómo & -0.132 \\
realizaron la tarea & $(1.072)$ \\
Plantea situaciones y/o problemas cotidianos & 0.832 \\
& $(2.039)$
\end{tabular}

Leer textos y realizar ejercicios de comprensión lectora

$6.175 * * *$

(1.910)

0.771

Les pide redactar pequeños textos literarios (cuentos, poemas, etc.)

(1.050)

Realiza dramatizaciones

$-1.616$

Acude a la biblioteca del establecimiento

(1.162)

$2.846 * * *$

(1.098)

Le preocupa la disciplina

$-2.597 *$

$-1.469$

(1.570)

(1.390)

Se preocupa de los que se atrasan y/o de que el curso entienda

1.218

0.937

(2.218)

(2.130)

Proporciona actividades más desafiantes a los más avanzados

1.890

$-0.096$

(1.318)

$(1.265)$

Se preocupa de que todos realicen actividades y/o los motiva

$-0.399$

$-2.049$

para que trabajen

(1.676)

(1.519)

Se preocupa de alcanzar a pasar todos los contenidos y/o

$-3.204$

$-0.488$

actividades

(2.160)

(2.021)

Da tareas del ramo más de 2 veces por semana

$-0.301$

$-0.104$

(1.191)

(1.090)

Cree que alumnos cursarán ed. superior

$5.844 * * *$

$5.850 * * *$

$(1.215)$

(1.131)

Está muy satisfecho con su trabajo

$-7.023$

$-4.844$

(5.697)

$(5.421)$

Grado de seguridad en sus conocimientos del ramo

$7.755 * *$

1.980

(3.112)

Constante

$105.220 * * *$

(2.980)

(11.907)

(11.261)

$\mathrm{R}^{2}$

0.110

0.132

$\mathrm{N}$

27515

28667

Notas:

*** Significativo al 1\%. ** Significativo al 5\%. * Significativo al 10\%.

Errores estándar ajustados por cluster a nivel de colegio.

La ecuación incluye además características de los alumnos y sus familias, del establecimiento y sus profesores. 
den a responder con mayor frecuencia que creen que sus alumnos cursarán educación superior. Al igual que en matemáticas, preocuparse por la disciplina tiene un efecto negativo y el grado de seguridad respecto de sus conocimientos es positivo, pero ninguno de ellos es significativo en términos estadísticos.

Algunos de estos resultados son consistentes con lo que muestran los estudios acerca de escuelas efectivas, en particular el que los profesores tengan altas expectativas de sus alumnos y la importancia de mantener la disciplina para así focalizarse sólo en el proceso de enseñanza-aprendizaje. Sin embargo, los resultados del análisis deben entenderse con cuidado, ya que como hemos mencionado se trata de correlaciones y no de relaciones causales.

Este análisis también se realizó diferenciando entre establecimientos educacionales urbanos y rurales y de acuerdo a la dependencia del establecimiento: municipal y particular subvencionado. Los resultados son similares a los obtenidos de la submuestra completa y se presentan en el Cuadro $N^{\circ}$ A1 del Anexo.

\section{Probabilidad de ser un buen profesor}

Con el objeto de profundizar en las prácticas de aula que se correlacionan con buenos resultados de aprendizaje, en esta subsección identificamos a los profesores que tienen un mayor SIMCE no explicado por las características de los niños y sus familias, de los establecimientos donde se educan y sus profesores.

De manera más específica, primero promediamos la parte no explicada del SIMCE de lenguaje y de matemáticas de los niños que están bajo la tutela de un mismo profesor. Es decir, asignamos a cada profesor los puntos promedio del SIMCE de cada área de su sala que el modelo de la ecuación (1) no puede explicar. La correlación entre este error promedio en matemáticas y lenguaje es de 0,74 , lo que sugiere que existen variables no observables, como la calidad del profesor, la motivación de los alumnos u otros, que llevan a que un buen resultado en un área del SIMCE esté acompañado por un buen resultado en la otra área también.

Luego generamos cuartiles de profesores desde los con menor desempeño no explicado hasta los con mayor desempeño no explicado, 
y estimamos un modelo logit que da cuenta de la probabilidad de ser un profesor que está en el cuartil (25\%) más alto; es decir, aquellos profesores que enseñan en cursos/colegios que muestran una mayor diferencia entre el valor efectivo del puntaje de la prueba SIMCE y el valor esperado, dadas las características de los niños, sus familias, el colegio y sus profesores. Las variables explicativas son las prácticas pedagógicas, preocupaciones y percepciones incluidas en el modelo de la subsección anterior.

El Cuadro $\mathrm{N}^{\mathrm{o}} 4$ muestra los resultados. Ellos son consistentes con los obtenidos al agregar las prácticas pedagógicas a la ecuación que explica el resultado de los niños en las pruebas SIMCE. Los profesores que se encuentran en el $25 \%$ superior en el caso de la prueba de matemáticas declaran en mayor medida ( 0,8 veces más que el resto) que piden a sus alumnos que expliquen a sus compañeros cómo hicieron sus tareas. También manifiestan con mayor frecuencia que plantean a sus alumnos problemas y situaciones relacionadas con la vida cotidiana (1,7 veces más). Asimismo, los buenos profesores declaran que creen que sus alumnos cursarán educación superior (1,4 veces más) y que se sienten seguros de conocer las materias que enseñan ( 1,9 veces más).

En el caso de lenguaje nuevamente se repiten las variables del análisis previo: los profesores que se encuentran en el $25 \%$ superior declaran que hacen a sus alumnos leer textos y ejercicios de comprensión lectora ( 1,8 veces más que el resto), que acuden a la biblioteca del colegio ( 1,4 veces más) y creen que sus alumnos asistirán a la educación superior (1,4 veces más). Adicionalmente, en este análisis surge una práctica que no había sido estadísticamente significativa anteriormente: el que el profesor pida a sus alumnos redactar pequeños textos literarios.

En el Cuadro $\mathrm{N}^{\circ} \mathrm{A} 2$ del Anexo se presenta este mismo análisis diferenciando entre establecimientos educacionales urbanos y rurales, y de acuerdo a la dependencia del establecimiento: municipal y particular subvencionado. Los resultados (con la excepción del caso de matemáticas para los establecimientos urbanos) son similares a los obtenidos en la submuestra completa. 
CUADRO No 4: PRÁCTICAS PEDAGÓGICAS DE LOS BUENOS DOCENTES

(Regresión logística, variable dependiente docentes en el 25\% superior)

\begin{tabular}{|c|c|c|}
\hline & Matemáticas & Lenguaje \\
\hline Desarrolla ejercicios de cálculo mental & $\begin{array}{c}0.910 \\
(0.109)\end{array}$ & \\
\hline Organiza competencias o juegos matemáticos entre los alumnos & $\begin{array}{c}1.142 \\
(0.142)\end{array}$ & \\
\hline $\begin{array}{l}\text { Pide a los alumnos que expliquen a otros compañeros cómo } \\
\text { realizaron la tarea }\end{array}$ & $\begin{array}{l}0.789 * * \\
(0.091)\end{array}$ & \\
\hline Plantea situaciones y/o problemas cotidianos & $\begin{array}{l}1.716^{* *} \\
(0.434)\end{array}$ & \\
\hline Leer textos y realizar ejercicios de comprensión lectora & & $\begin{array}{l}1.781 * * * \\
(0.445)\end{array}$ \\
\hline \multicolumn{2}{|l|}{ Les pide redactar pequeños textos literarios (cuentos, poemas, etc.) } & $\begin{array}{l}1.236 \\
(0.151)\end{array}$ \\
\hline Realiza dramatizaciones & & $\begin{array}{c}0.980 \\
(0.127)\end{array}$ \\
\hline Acude a la biblioteca del establecimiento & & $\begin{array}{l}1.375 * * * \\
(0.165)\end{array}$ \\
\hline Le preocupa la disciplina & $\begin{array}{c}0.834 \\
(0.146)\end{array}$ & $\begin{array}{c}0.824 \\
(0.141)\end{array}$ \\
\hline Se preocupa de los que se atrasan y/o de que el curso entienda & $\begin{array}{c}1.130 \\
(0.279)\end{array}$ & $\begin{array}{c}0.977 \\
(0.227)\end{array}$ \\
\hline Proporciona actividades más desafiantes a los más avanzados & $\begin{array}{l}1.171 \\
(0.166)\end{array}$ & $\begin{array}{l}1.102 \\
(0.151)\end{array}$ \\
\hline $\begin{array}{l}\text { Se preocupa de que todos realicen actividades y/o de motivar } \\
\text { para que trabajen }\end{array}$ & $\begin{array}{c}0.901 \\
(0.165)\end{array}$ & $\begin{array}{c}0.998 \\
(0.179)\end{array}$ \\
\hline $\begin{array}{l}\text { Se preocupa de alcanzar a pasar todos los contenidos y/o } \\
\text { actividades }\end{array}$ & $\begin{array}{c}0.702 \\
(0.194)\end{array}$ & $\begin{array}{c}1.013 \\
(0.251)\end{array}$ \\
\hline Da tareas del ramo más de 2 veces por semana & $\begin{array}{c}0.943 \\
(0.121)\end{array}$ & $\begin{array}{c}1.002 \\
(0.122)\end{array}$ \\
\hline Cree que alumnos cursarán ed. superior & $\begin{array}{l}1.409^{* * *} \\
(0.163)\end{array}$ & $\begin{array}{l}1.379 \text { *** } \\
(0.156)\end{array}$ \\
\hline Está muy satisfecho con su trabajo & $\begin{array}{c}0.651 \\
(0.513)\end{array}$ & $\begin{array}{l}1.045 \\
(0.708)\end{array}$ \\
\hline Grado de seguridad en sus conocimientos del ramo & $\begin{array}{l}1.943 * * \\
(0.625)\end{array}$ & $\begin{array}{c}0.617 \\
(0.195)\end{array}$ \\
\hline Pseudo $\mathrm{R}^{2}$ & 0.017 & 0.016 \\
\hline $\mathrm{N}$ & 1704 & 1769 \\
\hline
\end{tabular}

Notas:

*** Significativo al 1\%.** Significativo al 5\%.

Errores estándar ajustados por cluster a nivel de colegio.

Coeficientes en odds-ratio. 


\section{Conclusiones}

Este trabajo intenta medir el efecto de las características observables de los docentes sobre el resultado de los escolares en la prueba SIMCE de cuarto básico del año 2002.

En la primera parte del trabajo describimos los rasgos generales de los profesores en Chile en todos los niveles del sistema escolar. El análisis revela que, al igual que en otros países, los profesores son en su mayoría mujeres. Asimismo, los datos muestran que la mayor parte posee un título profesional en educación y que un amplio porcentaje tiene más de 20 años de experiencia en el aula.

En la segunda parte del estudio explicamos las diferencias en el desempeño de los niños en el SIMCE 2002 en base a la variabilidad observada en sus propias características y en las de sus familias, los establecimientos y los profesores que los educan. Para corregir por sesgos en la asignación de profesores a través de escuelas, controlamos por el desempeño de los establecimientos en pruebas SIMCE anteriores. A su vez, para corregir por sesgos en la asignación de profesores al interior de las escuelas, restringimos la muestra a establecimientos que tienen una sola sala en el nivel. Nuestros resultados indican que en la prueba de lenguaje —no así en matemáticas—, los alumnos educados por docentes mujeres obtienen mejores resultados en el SIMCE que aquellos educados por docentes hombres. Asimismo, en ambas pruebas existe correlación entre el desempeño escolar y el hecho de que el profesor tenga un título docente, aunque el tipo de institución —universidad o escuela normal- que confirió el título sólo es relevante en la prueba de lenguaje. Este efecto es económicamente relevante: los alumnos cuyos docentes poseen el título de profesor obtienen un puntaje adicional de hasta $13 \%$ de una desviación estándar. Finalmente, el efecto de los años de experiencia docente es no lineal, fundamentalmente en lenguaje, con las mayores ganancias en los primeros años de carrera profesional, resultado coincidente con el de investigaciones para otros países.

En el trabajo también se analizan las prácticas que los profesores declaran realizar en su labor docente, las preocupaciones que éstos declaran y el grado de satisfacción con su trabajo. Nuestro análisis revela que prácticas tan simples como leer textos y realizar ejercicios de com- 
prensión lectora y llevar a los niños a la biblioteca se correlacionan con un mejor desempeño en el SIMCE de lenguaje. Además, aquellos profesores que declaran creer que sus alumnos cursarán educación superior obtienen un puntaje adicional de un $12 \%$ de una desviación estándar en ambas pruebas SIMCE.

Por su parte, los profesores de las salas de mejor desempeño tienden a declarar con mayor frecuencia que creen que sus alumnos cursarán educación superior, y que desarrollan actividades como, por ejemplo, pedir a los alumnos que expliquen a sus compañeros cómo realizaron las tareas y plantear situaciones y problemas cotidianos en matemáticas, y acudir a la biblioteca y realizar ejercicios de comprensión lectora en lenguaje.

Estos resultados, sin embargo, deben leerse con cuidado, debido a que estas prácticas pedagógicas simplemente pueden estar correlacionadas con otras variables no observables. Es posible, asimismo, que la causalidad sea inversa, de modo que niños con buenas características no observables pueden permitir un mejor desarrollo de la clase, favoreciendo la realización de ciertas actividades docentes.

Nuestros resultados son consistentes con los obtenidos en estudios realizados para otros países con mejores datos, principalmente los Estados Unidos, que permiten comparar el desempeño de los mismos estudiantes con diferentes profesores, y de esta forma distinguir la variación de la calidad de los profesores de la variación de la capacidad y habilidad de los estudiantes. En particular, estos estudios encuentran que la experiencia docente tiene un impacto significativo en los resultados de los estudiantes y que las mayores ganancias debido a la experiencia suceden en los primeros años de la carrera docente.

Asimismo, los resultados encontrados respecto de las prácticas pedagógicas que tienen alguna correlación con los resultados de los alumnos en la prueba SIMCE son consistentes con lo que encontramos en un estudio acerca de la Sociedad de Instrucción Primaria (SIP), el que buscaba comprender las razones del éxito académico de estos colegios. En particular, todos los directores de la SIP entrevistados mencionaban como prácticas sistemáticas de sus profesores, entre otras, visitas periódicas a la biblioteca. También mencionaban la importancia de que los profesores creyeran en la capacidad de sus alumnos y tuvieran altas expectativas acerca de su futuro (Henríquez et al. 2009). 


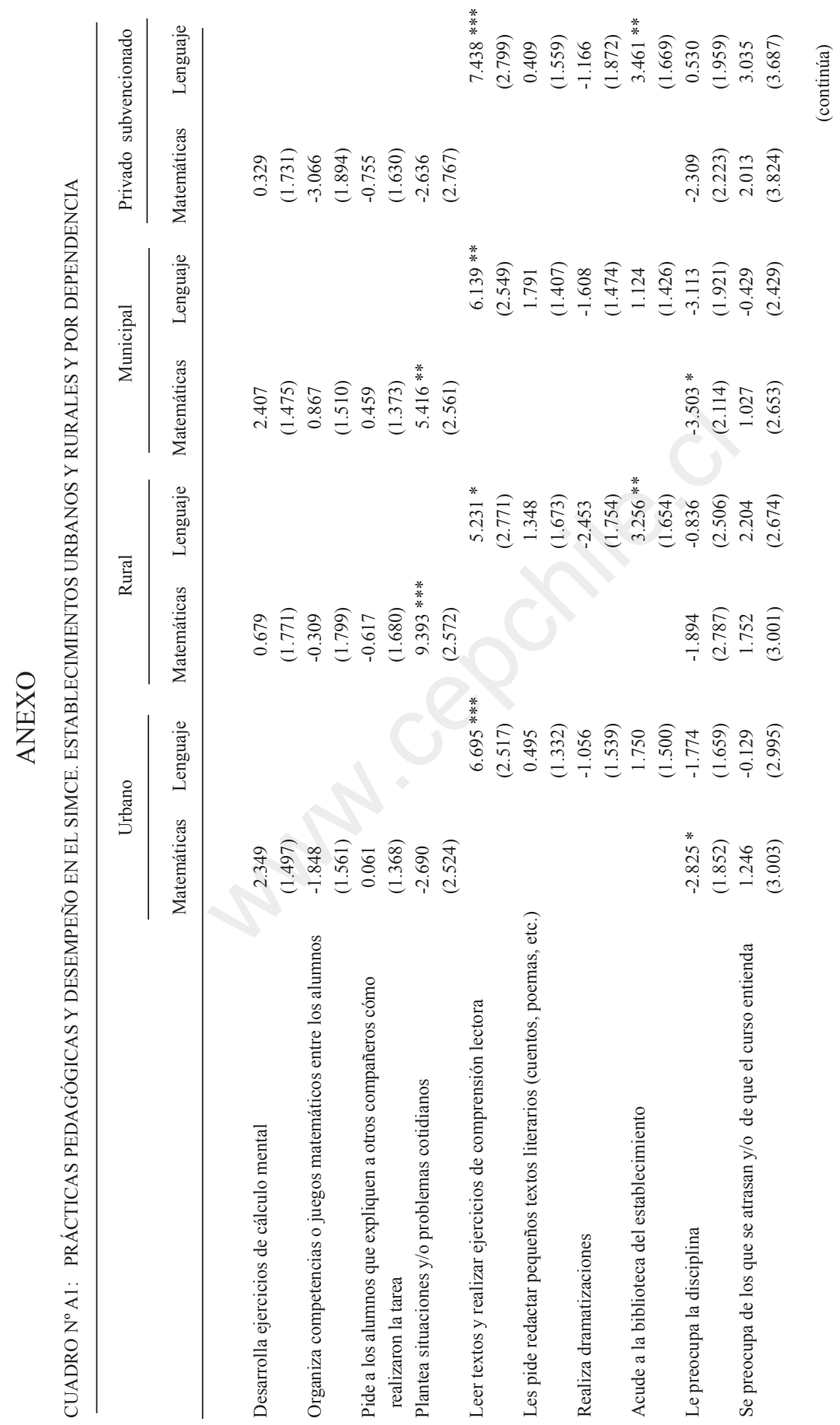




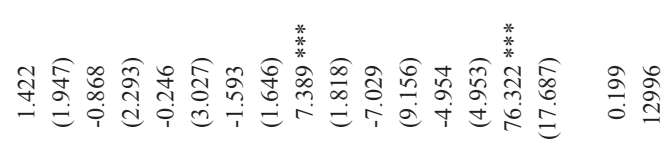

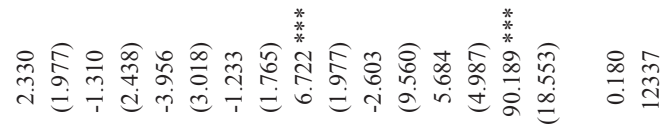

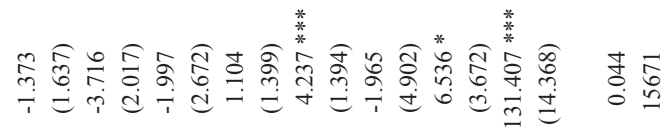

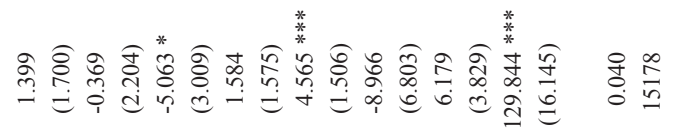

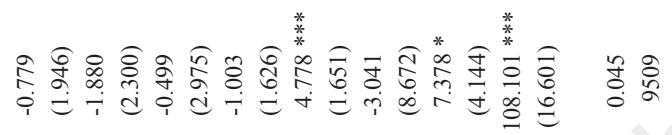

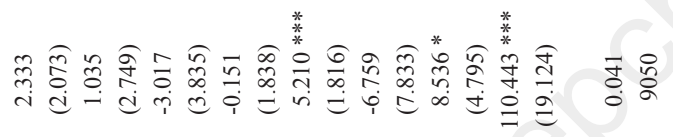

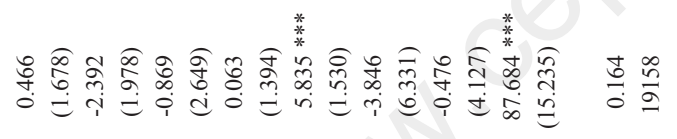

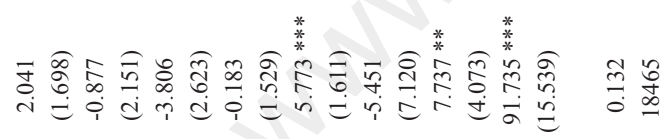

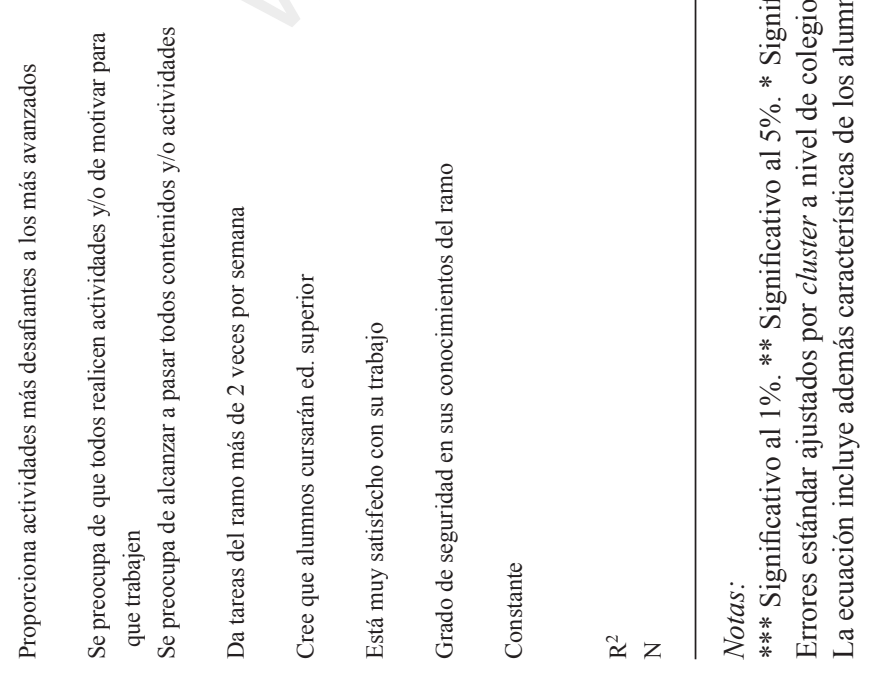




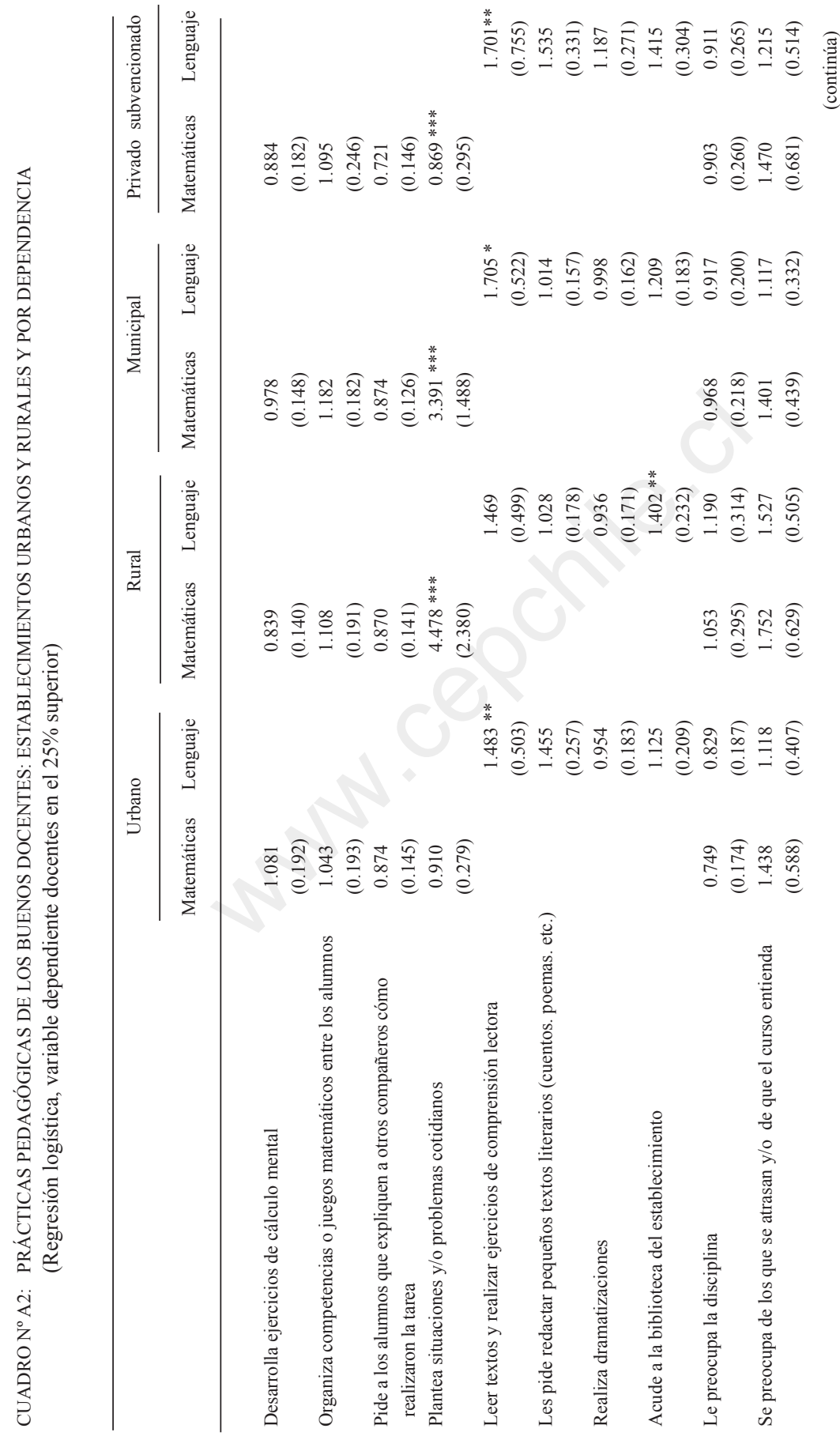




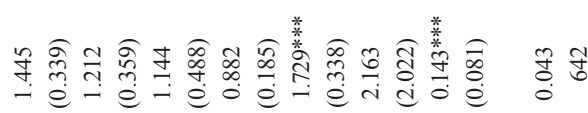

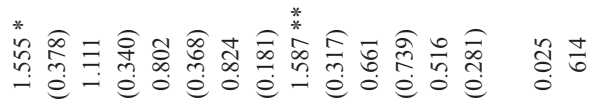

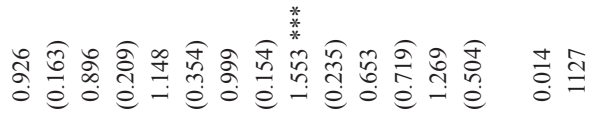

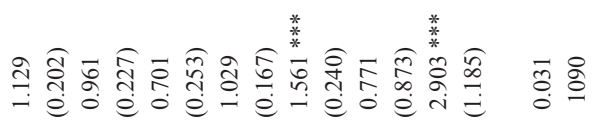

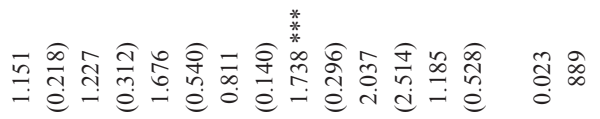

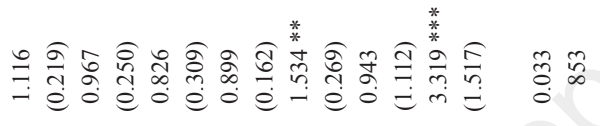

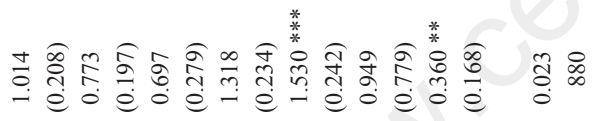

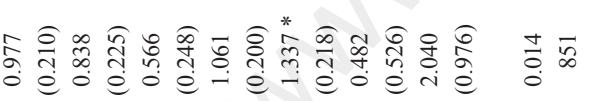

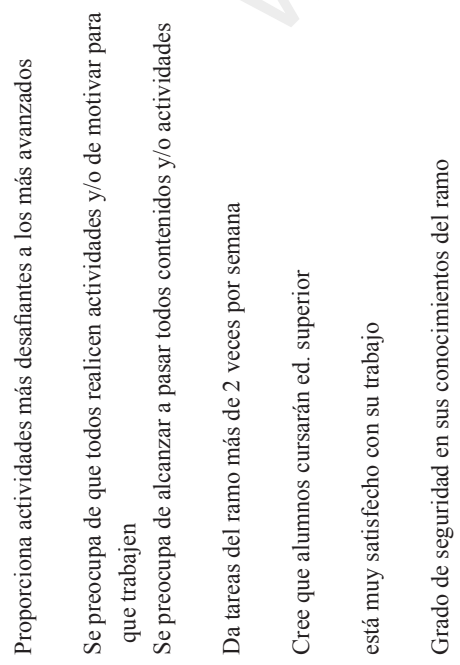

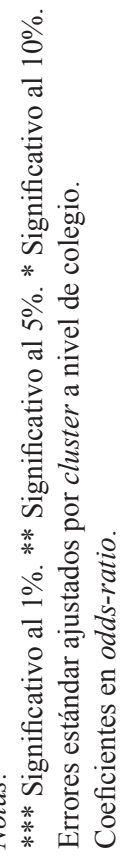




\section{REFERENCIAS}

Bravo, D., D. Falck, R. González, J. Manzi y C. Peirano. "La Relación entre la Evaluación Docente y el Rendimiento de los Alumnos: Evidencia Para el Caso de Chile". Manuscrito Centro de Microdatos, Universidad de Chile, 2008.

Clotfelter, T., H. F. Ladd y J. L.Vigdor. "Teacher-Student Matching and the Assessment of Teacher Effectiveness". The Journal of Human Resources, Vol. 41, № 4, pp. 778820, 2006a.

- "How and Why do Teacher Credentials Matter for Student Achievement?" Mimeo, Duke University, 2006b.

- "Teacher Credentials and Student Achievement: Longitudinal Analysis with Student Fixed Effects". Economics of Education Review, Vol. 26, № 6, pp. 673682, 2007.

Coleman, J., E. Campbell, C. Hobson, J. McPartland, A. Mood, F. Weinfeld y R. York. Equality of Educational Opportunity. U.S. Government Printing Office, 1966.

Gallego, F. y A. Hernando. "School Choice in Chile: Looking at the Demand Side". Documento de Trabajo 356, Universidad Católica de Chile, 2009.

Goldhaber, D. D. y D. J. Brewer. "Does Teacher Certification Matter? High School Teacher Certification Status and Student Achievement". Educational Evaluation and Policy Analysis, Vol. 22, N² 2, pp. 129-145, 2000.

Hanushek, E. A. "Assessing the Effects of School Resources on Student Performance: An Update". Educational Evaluation and Policy Analysis 19(2): 141-164, 1997.

Hanushek, E., J. Kain, D. O’Brien y S. Rivkin. "The Market for Teacher Quality". NBER Working Paper No 11154, 2005.

Henríquez, F., B. Lara, A. Mizala y A. Repetto. "Effective Schools Do Exist: Low Income Children's Academic Performance in Chile". Documento de Trabajo CEA, 2009.

Kukla-Acevedo, S. "Do Teacher Characteristics Matter? New Results on the Effects of Teacher Preparation on Student Achievement". Economics of Education Review 28: 49-57, 2009.

Lara, B., A. Mizala y A. Repetto. "The Effectiveness of Private Education: Evidence from Structural School Switches". Documento de Trabajo CEA, 2009.

León, M. G., J. Manzi y R. Paredes. "Calidad Docente y Rendimiento Escolar en Chile: Evaluando la Evaluación”. Manuscrito, Universidad Católica de Chile, 2009.

Mizala, A. y B. Schneider. "Negotiating Education Reform: Teacher Evaluations and Incentives in Chile after 1990". Mimeo, Universidad de Chile.

Rivkin, S., E. Hanushek y J. Kain. "Teachers, Schools and Academic Achievement". Econometrica, Vol. 73, № 2, 2005.

Rockoff, J. "The Impact of Individual Teachers on Student Achievement: Evidence from Panel Data". American Economic Review 94(2): 247-252, mayo, 2004.

Toledo, G. "Calidad Docente y Logro Escolar: Enfrentando el Problema de Ordenamiento no Aleatorio entre Características de Profesores y Alumnos". Tesis de Magíster en Economía, Universidad de Chile, 2010.. 